\title{
Beyond Consciousness to Cosmos-Beyond Relativity and Quantum Theory to Cosmic Theory
}

\author{
Henry H. Lindner
}

\begin{abstract}
Albert Einstein was a subjectivistic mathematical idealist. His physics consisted of mathematical models of subjects' ideas-their sensations and measurements. Einstein's "objective reality" was intersubjective reality - the experiences about which various observers could agree. His "causes" were mental constructs created to systematize the observers' experiences. He modeled reality as it was experienced and thought, not as it was. He modeled consciousness, not the Cosmos. If we want to understand the Cosmos as it is, as a physical system from which we and our consciousness evolved, we must build upon a different philosophical foundation. We must create hypotheses and theories about what exists and how it interacts with our minds and our instruments. We must reàch beyond consciousness to Cosmos, beyond Relativity and Quantum Theory to Cosmic theory.
\end{abstract}

Key words: cosmism, Cosmos, epistemology, ether, idealism, light, logic, mass-energy, mathematics, metaphysics, motion, objectivism, paradox, philosophy, photon, Quantum Electrodynamics, Quantum Mechanics, Quantum Theory, Relativity, space, space-time, subjectivism, theory, time

\section{INTRODUCTION}

If we accept the theory that a physical Cosmos exists, and that our sensations and measurements result from our interaction with the Cosmos, then we should not restrict our physics, as Albert Einstein did, to the modeling of our sensations and measurements. We should theorize about this Cosmos-about the physical substance(s) whose alterations, motions, and evolution have produced matter, electromagnetism, gravity, and life. No matter how accurately Relativity and Quantum Theory (QT) ${ }^{1}$ model the facts of experience, no matter how useful they may be, they are neither models of the Cosmos nor theories of the Cosmic entities and processes that produce our experiences. Einstein created both models in 1905 using the idealistic metaphysics and subjectivistic epistemology that he inherited from Ockham, Descartes, Locke, Berkeley, Hume, Kant, and Mach. His absolute $c$, time dilation, space-time, mass-energy, and photon are not physical hypotheses, they are "freely created" concepts for the limited purpose of "finding regulative connections between our sensual experiences." ${ }^{(1)}$ Relativity and QT thus relate all events, such as trajectories, mass, and the velocity of light, to the observers' frames or to arbitrarily chosen coordinate systems (CSs) instead of to the matter and space of the Cosmos. They treat light/matter interactions as probabilities of the occurrence of observable "events" instead of creating a coherent physical theory of what light is, what matter is, and how they interact so as to produce the events that we can observe. Einstein's models obviously contain many true ideas and working equations; these exist, however, within an esoteric ideological framework that few people understand.
Physicists and philosophers err in believing that they can use Einstein's idealistic concepts and models to explain Cosmic reality. Since they were not designed for that purpose, the result is contradiction and confusion (the light paradox, the twin paradox, the inertia paradox, the photonic paradoxes, Schrödinger's Cat, the measurement problem, etc.). Relativists and Quantum theorists try to "resolve" these paradoxes with a familiar host of apologetic rationalizations and ad hoc fixes. Another problem is that these two subjectivistic models are incompatible - they share neither a conceptual nor a mathematical foundation. Relativity is a deterministic model of an underlying space-time matrix that determines (represents) the observers' measurements of distance and time. Quantum Mechanics (QM), evolved from Einstein's QT, is instead a probabilistic model of the observers' experiences of quantized light/matter interactionsevents that neither are related to any underlying "reality" nor have any dependence on the observers' frames or motion. It should thus be no surprise that, in order to create a coherent theory of the Cosmos, both of these models must go, along with their esoteric metaphysics and epistemology. Every true idea they contain must be recycled for use within a coherent philosophical program - an evolutionary physical Cosmology.

In this paper, I will demonstrate that Relativity and QT are manifestations of an obsolete, pre-Darwinian theory of the mind and its relationship to its objects, and I will assert a new metaphysics and epistemology for physics that are consistent with the evidence, the best theories, and the actual beliefs of most physicists. 


\section{DEFINITIONS}

Please bear with me while I present some crucial definitions; without them, everything I say about Einstein's work can be misunderstood and misconstrued. This is a difficult philosophical problem and, like most such problems, it persists and appears irresolvable because it is mired in ambiguous terminology. To eliminate this problem in this case, I must expose the central issue that "modern philosophy" has evaded for several centuries. I must expose the philosophical bedrock - the two underlying, mutually exclusive theories about what exists, how it relates to our conscious experience, and how we should study it:

idealism: Consciousness exists. All that we know with certainty is that we are conscious-we passively experience and actively create ideas. The existence of anything apart from our ideas is uncertain and unknowable.

Cosmism: The Cosmos exists. It is the self-sustaining physical entity of whose evolution we humans and our language-enhanced consciousness are products. Our sensations and measurements result from the physical interaction of our sense organs and instruments with the rest of the Cosmos.

These mutually exclusive metaphysical postulates produce different epistemologies:

subjectivism: Knowledge consists only of our descriptions and models of our conscious experiences.

objectivism: Knowledge consists of our attempts to describe, model, and explain the cosmos itself, as it exists and evolves and causes our experiences.

These metaphysical/epistemological alternatives have been called the "primacy of consciousness" and the "primacy of existence." (2) Idealism holds that our minds participate in a mental/spiritual reality that is separate from and independent of any physical, biological, or neurological reality. It has produced subjectivism, skepticism, spiritualism, mentalism, empiricism, phenomenalism, and positivism. Cosmism, on the other hand, asserts that everything, including our language-enhanced consciousness, is a product of the evolution of the Cosmos. Cosmism has produced objectivism, realism, naturalism, materialism, and evolutionary theory.

In theoretical physics, these two distinct theories of reality and its relationship to our conscious experience prescribe two different ways to acquire concrete factual knowledge:

subjectivistic description: The observer merely describes his experiences-what he senses with and without instruments. Since no physical Cosmos exists or can be known, he can only relate these events to his own frame of reference or CS.

objectivistic description: The observer posits a Cosmos and Cosmic entities and CSs that play a unique and causal role in what she experiences. She relates her measurements and experienced events to these Cosmic CSs.
We are curious creatures. Not content to merely describe things, we construct models that can account for the regularities we observe. There are again just two approaches:

subjectivistic modeling: Regularities are noted among the observed events and measurements in the subject's CS. The observer then constructs a system of assumptions about what he measures and, using this system, finds the "laws" and equations that fit his observations and successfully predict future observations. The result is a model of the entities and events in the observer's experiential "world" or CS.

objectivistic modeling: Regularities are noted among events and measurements as these are related to Cosmic entities and CSs. The observer finds the "laws" and equations that describe motion in the Cosmic CSs and that predict future observations. The result is a model of the Cosmos.

Idealism and subjectivism have given us a physics limited to subjectivistic modeling:

Relativity: All motion is merely relative to, and therefore equally well related to, any observer or arbitrarily chosen CS. The "laws" of physics are the mathematical correlations of measurements made by any observers in any CSs. The fundamental entities, mass-energy and space-time, are observers' measurements. A physical Cosmos that causes the observers' experiences or affects the observers' instruments is a superfluous hypothesis.

Quantum Theory: The "laws" of physics are merely descriptions of our experiences-mathematical instruments for predicting the probability that one set of observations will be followed by a certain second set of observations. Sources produce neither physical waves nor particles, but only probabilities that an observed event will produce a subsequent observed event in a certain location at a certain time. Consciousness is the only reality. There is no underlying physical reality to describe or explain.

Cosmism and objectivism, however, allow additional levels of investigation and cognition:

Cosmic theory: The attempt to explain our existence and experiences as caused by observed and unobserved entities and processes.

Cosmic philosophy (natural, scientific): The creation and criticism of theories to explain all phenomena, the practical applications of these theories, and the mental tools and techniques required for these tasks.

Allow me to illustrate the above definitions with the history of theoretical physics. The Aristotelian and Ptolemaic geocentric system was an objectivistic model of the Cosmos-an attempt to model what existed prior to and apart from any observer. However, it naively presumed that the observer's accidental frame, Earth, was motionless in the center of the Cosmos. This system required the rotation of the 
rest of the Cosmos and needed many complicated devices to model the planets' motions. To make sense of the Cosmos as a physical system, Copernicus posited instead that Earth rotated and moved about the Sun, even though humans could not directly sense or measure these motions. This required a cognitive leap from the subject's experience in her own CS to an objectivistic model of motion in a Cosmic CS-a theory of what was happening in the Cosmos to produce the subject's experiences-a leap from the known (experience) to the unknown (Cosmos). Opponents argued that this leap was unjustified because observers experienced no such motions-they felt no spatial "wind." However, we know that Copernicus was correct and that all subsequent advances in Cosmology and physics required this shift from the Earth CS to the Sun-Star CS. It enabled Kepler to discover the elliptical shape of the orbits, which enabled Newton to demonstrate that the motion of our Moon and planets was caused by the same gravity that we experience on Earth. Newton's physics was also objectivistic in that it related all motion not to the human observer's CS but to a Cosmic CS. His absolute space was a static, Euclidean, universal ether that resisted the acceleration of matter and in which all matter had some definite velocity, even if it could not be determined. ${ }^{(3)}$ James Maxwell added to this ether the property of propagating electromagnetic (EM) waves at $c$. Michelson and Morley $(M-M)$ then tried to detect the velocity of the Earth through it using the interference of light waves traveling in different directions. They sought the very same "wind" assumed by Copernicus's opponents. When they failed to detect it, the Copernican debate was resurrected: Is Earth really moving through Cosmic space? Stokes responded to the null M-M results with the theory that Earth entrained its surrounding space into its own motion-that the spatial ether was not static but some kind of gas or fluid whose position and motion were dynamically influenced by matter. Hendrick Lorentz tried to save Newton's static universal space by theorizing that the null $M-M$ results were an illusion caused by the effects of static space on the moving apparatus. He proposed that objects moving through the ether suffered a physical length contraction and reduction in frequency that made the detection of absolute motion impossible. He produced equations to describe these alterations. The possible objectivistic explanations of the null $M-M$ results were in no way exhausted. Einstein, however, attempted to implement a completely different metaphysical and epistemological program. He concluded that the null $M-M$ experiments implied that there was no physical space through which Earth was moving - that Earth was not "really" moving at all. He asserted that the ether hypothesis was "superfluous" and that all motion was merely relative-the trivial consequence of one's choice of CS. He reproduced Lorentz's ether equations but applied them only to the comoving CSs. He thus eliminated the middle ethereal term and any possible physical explanation of these effects. He related all motion and laws of motion to any observer's or any chosen CS, not to the matter and space of the Cosmos. He attempted only to find the simplest set of ideas that would describe and predict the observer's experiences and measurements. He thus abandoned objectivism and physical theory in favor of subjectivistic modeling. His 1905 papers on Relativity and the light quantum put the existence of the physical cosmos, its physical substance( $(s)$, and the causes of all physical phenomena outside the purview of Science. Einstein claimed that he had reversed the Copernican revolution-that Relativity made the historic struggle between the views of Ptolemy and Copernicus "meaningless" since it was only a matter of one's choice of CS. ${ }^{(4)}$ In fact, if we want to model and to understand the Cosmos, we must again take the Copernican leap from observations to Cosmos.

I know that some readers will protest that Relativity and QT are not "subjectivistic" because they deal only with facts and measurements and have no element of personal whim or bias. However, the "subjectivity" they speak of is not the "subjectivism" of philosophical parlance defined above. I do not charge Relativity and QT with a naive psychological or perceptual subjectivity. Instead, epistemological subjectivism assumes the observer's accurate accounting of his experiences and measurements, including instrumented measurements, within his CS, even as recorded by mini-observers at every point of his CS. The argument is thus an equivocation-an attempt to confuse two different concepts.

\section{A BRIEF HISTORY OF SUBJECTIVISM}

Why did Einstein resort to subjectivistic modeling? How did this highly restricted epistemology come to dominate physics? Why don't we abandon his approach and instead create objectivistic models and physical theories of Cosmic space, matter, and motion? These questions must be answered at two levels: the practical and the ideological. Practically, in the wake of the null $M-M$ experiments, scientists were understandably dissatisfied with the Newtonian/Lorentzian theory of a single static ethereal space, but had none of the information or tools needed to create a working dynamic theory of space, light, and gravity. They did not know what we know today about the behavior of light, atoms, and atomic clocks at rest or in motion among the celestial bodies of this Cosmos. Theoretical physics was at an impasse. Einstein cut this Gordian knot by abandoning objectivism and causal theory altogether. He greatly simplified physics by dealing only with appearances and measurements, thus evading the difficult questions of the causes of Cosmic phenomena. Why did most other scientists eventually accept this approach? Why does it persist today? To answer the ideological question, we must briefly review the history of Cosmic philosophy and of its nemeses-idealism and subjectivism. We will then discuss the application of these ideas in Special Relativity (SR), General Relativity (GR), and QT.

Philosophy originated in Ionia in the 6th century B.C. when Thales of Miletus theorized that all physical entities and processes were the result of changes in and of water. This historic conjecture replaced traditional mythological Cosmol- 
ogies with naturalistic theory. It began a program of openended, nondogmatic theorizing about the nature of the Cosmos and of the causes of things. Thales's own colleagues posited other substances as fundamental to the cosmos. Later, on the Greek mainland, Leucippus and Democritus theorized that the Cosmos and all its manifestations were the result of invisible atoms flying through a void. Of course, these early theorists had little of the knowledge we have today. Their theories were thus inadequate and full of difficulties.

Socrates' early interest in Cosmic theory gave way to a disappointed skepticism. The problems he encountered in such theories made him doubt the efficacy of all materialistic explanations and even of his own senses. He decided to "take recourse to the world of mind and seek there the truth of existence." ${ }^{(5)}$ Aristippus, a follower of Socrates, developed Socrates skepticism. His Cyrenaic school rejected Cosmic theory, arguing that humans could only know their own sensations and experiences and could not have any knowledge of the external world. Protagoras, the earliest known sophist, argued further that "Man is the criterion of all objects, of those which exist that they exist, and of those that exist not that they exist not." Of him it was said "he posits only what appears to each individual, and thus he introduces relativity. ${ }^{\prime(6)}$ Aristotle opposed the skepticism, subjectivism, and relativism of these thinkers with many arguments:

For sensation is surely not the sensation of itself, but there is something beyond the sensation, which must be prior to the sensation; for that which moves is prior in nature to that which is moved... (7)

He demonstrated that subjectivism produces contradiction since it cannot account for illusions and because subjects must always disagree on what they experience and measure. In another passage, he states,

But if not all things are relative, but some are self-existent, not everything that appears will be true; for that which appears is apparent to someone; so that he who says all things that appear are true, makes all things relative. ${ }^{(8)}$

Aristotle argued that gravity was proof that all motion was not merely relative, as objects gravitated Earthward regardless of the position of the observer or the choice of frame, ${ }^{(9)}$ an argument no less cogent today. Later, Isaac Newton also rejected subjectivism in physics:

But because the parts of space cannot be seen, or distinguished from one another by our senses, therefore in their stead we use sensible measures of them ... And so, instead of absolute spaces and motions, we use relative ones; and that without any inconvenience in common affairs; but in philosophical disquisitions, we ought to abstract from our senses and consider things themselves, distinct from what are only sensible measures of them. ${ }^{(10)}$
Aristotelian philosophy and Christianity were married in the Dark Ages, but with the discovery of Aristotle's works of natural philosophy, churchmen perceived a threat to their beliefs and the marriage became strained. Thomas Aquinas attempted to reconcile the partners, but could not prevent the inevitable divorce. In 1277, just three years after Thomas's death, the Bishop of Paris issued a condemnation of many Aristotelian-Thomist theses. This divorce was accompanied by a religious backlash against Aristotle and all natural philosophy. William of Ockham (b. 1280) tried to completely free theology from Cosmic philosophy. To do so, he limited philosophy to the mere description of experience. Against physical causation and evolution, he stressed the omnipotence of God and the radical dependence of all created things upon God for both their existence and interaction. For Ockham, there was a created world, but there were no Godindependent physical entities or processes. Therefore, to study nature was to study God's free acts. Ockham therefore insisted that we should only describe our actual experiences using the fewest propositions necessary; we should only note the regular sequences of observed events, not hypothesize about any God-independent natural causes or mechanisms. For Ockham, experience-based propositions, not Cosmic entities and processes, were the proper objects of knowledge. ${ }^{(11)}$ The purpose of Ockham's Razor was clear-to eliminate all complex, hypothetical theories of physical entities and causation in favor of the simplest explanation of the cause of all experienced phenomena-God.

René Descartes went one step further toward spiritual subjectivism. He sought to confine knowledge to what was given to us by God and about which we could be certain-the "clear and distinct ideas presented to the senses." His Cogito ergo sum, "I think, therefore I am," tacitly assumed idealism-the primacy of consciousness-that consciousness existed prior to, and independent of, the human body and the physical Cosmos. The apparent Cosmos could, he supposed, be a hallucination produced in his mind by some demon. ${ }^{(12)}$ Descartes was persuaded to believe in the existence of the physical cosmos because it appeared to exist and he believed that God would not so deceive him if it were not so. However, he believed in "external" things only insofar as they were manifestations of geometrical and mathematical forms. In order to avoid error, Descartes asserted that we must shun all theorizing about physical entities and causes and confine ourselves to mathematical descriptions. He implied that theorizing about the final (Cosmic) causes of things was a $\sin .{ }^{\{1\}}$ He advised that we pursue the only certain, indubitable knowledge available-the mathematical description of our sensations-universal mathematics. To this purpose, Descartes invented the familiar three-axis CS that represents space as a three-dimensional geometric matrix.

John Locke was also more certain of spiritual than of physical existence. ${ }^{(14)}$ He claimed that we could only know the ideas that were presented to us by our senses and could never pass beyond them to any knowledge of the nature or hidden causes of things. ${ }^{(15)}$ Descartes and Locke were thus 
dualists, believing in the existence of two substancesspiritual and physical. Since they knew very little about physical reality or the human mind, they could not produce a good theory of how these two kinds of reality coexisted and interacted. They could not explain what matter was or how it interacted with "spirit." They therefore defaulted to the simpler, immediately accessible, conscious/spiritual perspective (idealism-subjectivism) and tried to discourage all theorizing about the unseen physical causes of phenomena.

Bishop Berkeley (circa 1710) completed the move toward idealism and subjectivism and away from Cosmic philosophy. In his post-Copernican, post-Newtonian world, he feared that as more and more phenomena were explained by natural causes acting in a physical Cosmos, faith in the God of Christianity would gradually give way to materialistic natural philosophy (and this was before Darwin). He saw all philosophical explanation as a slippery slope leading to atheism, so he tried to replace Cosmic philosophy with a purely descriptive approach to knowledge that had no need for the Cosmos or for physical causation. He rejected Descartes and Locke's dualism because it admitted the existence of a physical Cosmos and thus left the door open to natural philosophy. He argued instead that since we humans know only our own conscious experiences and have no direct knowledge of any material Cosmos, we must not assume that it exists. ${ }^{(16)}$ He argued that we should accept the simpler theory that all reality is purely spiritual; ${ }^{(17)}$ that our experiences are not caused by any physical world but are a virtual reality provided directly to our (disembodied) minds by God ${ }^{(18)}$ Berkeley argued, for instance, that people would not worship the Sun once they realized that it was not an independently existing physical entity, but merely a Godgiven apparition. ${ }^{(19)}$ Berkeley asserted that the laws of optics were God's means of creating the visual illusion of distance in our hallucinatory consciousness, an illusion properly coordinated with illusions of our own motion and action within this virtual space. ${ }^{(20)}$ (An analogue of Berkeley's theory of consciousness was recently presented in the film, The Matrix. ${ }^{(21)}$ In it, humans believe that they are experiencing normal life in the physical world when in reality their bodies are lying in vats, their brains are hardwired to a master computer, and their minds are interacting with each other's within a computer-generated virtual reality. Berkeley's Matrix likewise consisted of individual human spirits interacting with each other within a God-generated spiritual reality.)

Berkeley publicly accused Newton of advocating an atheistic theory, because Newton's absolute space, absolute time, and physical matter were supposed to exist "without the mind" and thus without God. ${ }^{(22)}$ Echoing Ockham, Berkeley argued that since God alone makes one experience follow another, philosophy should merely note these regularities and not pretend to explain things by corporeal causes. ${ }^{(23)}$ He argued that gravity was not an essential quality inherent in bodies but was simply a motion entirely dependent on the will of God-who causes some bodies to tend toward each other, others to stay at fixed distances, and others to fly asunder as He sees convenient. ${ }^{(24)}$ Against Newton, he argued that we should treat motion not as absolute, Cosmic, or real but as merely relative to ourselves and to any other objects in our sensoria, ${ }^{(25)}$ and treat time as the mere succession of events in our consciousness. ${ }^{(26)}$

Following Berkeley in this "Enlightenment," David Hume denied that we could know any Cosmic causes, saying that our belief that an effect will follow from a cause is merely a "custom" we have acquired through repetitive experience. (27) He denied that we could ever know the cause of such a "custom" as gravity. ${ }^{(28)}$ Like Berkeley, he reduced the Cosmos and its causes to correlations among experiences. Immanuel Kant agreed that we could only know the phenomena presented to our senses and could never pass beyond them to any knowledge of the noumena-things in themselves. Kant claimed that the mind imposed its own order on phenomena using a priori categories like space, time, and causality. These categories were ideals that were not inductively gained from experience but were innate mental structures.

Ernst Mach admitted his debt to Berkeley and Hume. ${ }^{(29)}$ He stated, "The world consists only of our sensations." ${ }^{(30)} \mathrm{He}$ asserted that the aim of Science was to describe our sensations and that the laws of Science merely stated general relationships among our sensations. Anything not directly perceived did not exist; unapparent entities posited to cause sensations were mere "metaphysical speculations" -nothing more than convenient fictions. Therefore, Mach, as late as the early 20th century, denied the existence of atoms because they could not be seen, even though they were a necessary postulate in any attempt to explain our experiences. Mach redefined physical theories as mere quantitative instruments-mathematical models for facilitating the mental reproduction and prediction of facts. Mach wrote extensively and greatly influenced the scientists and philosophers of the late 19th and early 20th centuries. His idealist ideology, under the names "sensationalism," "phenomenalism," and "positivism," was considered the modern and enlightened approach to Science at the turn of the last century. In 1883, Mach followed Berkeley in branding Newton's theory of absolute space and motion as "devoid of content," saying that we have knowledge only of relative spaces and motions." (31) He called those who shared his view "relativists." (32)

Albert Einstein had a lifelong interest in philosophy-he had read Kant as a boy, he had read Berkeley, and he admitted that Hume and Mach influenced his development. ${ }^{(33)}$ So we can understand why he asserted,

The only justification for our concepts and system of concepts is that they serve to represent the complex of experiences; beyond this they have no legitimacy. ${ }^{(34)}$

Following Berkeley, Einstein eschewed Cosmic theory and natural philosophy in favor of descriptive Science, which he 
regarded as "methodical thinking directed toward finding regulative connections between our sensual experiences."(35) Einstein shared Berkeley's belief in God as "a superior mind that reveals itself in the world of experience."(36) Like Descartes, Einstein was a mathematical idealist-believing that "nature is the realization of the simplest conceivable mathematical ideas."(37) He sought, therefore, to grasp the mathematical ideas by which God created our experiences by discovering the fewest and simplest "laws" that could account for them. Following Berkeley, Einstein defined the "real external world" intersubjectively, as the sum total of all experienced events about which subjects can agree. ${ }^{(38)}$ Following Berkeley and Mach, Einstein initially refused to believe that space itself had any physical qualities because it was not directly evident to our senses or instruments.

Einstein's Relativity and the QT were thus faithful implementations of Bishop Berkeley's vision of Science. They merely described the observer's experience with no reference to any physical Cosmos or causes-as if humans were experiencing a shared hallucination and wanted only to discover its rules. These models' only reality was consciousness and its contents. They dealt with the observers' "information," not with the Cosmos. Their "laws of physics" were just mathematical correlations of the observers' measurements, their "space" a number of the observers' measuring rods, their "time" a number of ticks of the observers" clocks, their "mass" a measured or calculated quantity, and their "light" just a probability that a future quantized event will be observed. The foundations of Einstein's model of reality, "mass-energy" and "space-time," were nothing but quantities and geometries invented by the observer to model the mathematical order behind his measurements. Thus we are saddled today with a theoretical physics that does not and cannot theorize about the cosmos or the causes of physical phenomena-a physics based upon a spiritualistic theory of the relationship of our minds to the Cosmos.

\section{EINSTEIN'S SUBJECTIVISTIC MODELS}

Since I've leveled serious charges against Einstein's physics, it behooves me to support and explain them in some detail. Since Einstein began his career as a Machian sensationalist, I will address the subjectivism of his models first. Later, I will deal with the problem of the inappropriate reification of his idealistic constructs.

\subsection{Special Relativity}

Let us look more closely at Relativity. Einstein chose, as the first axiom and foundation of his system, Galileo's own principle of relativity. This principle encompassed the fact that the rules of ballistics were similar for any observer moving parallel to Earth's surface at (subluminal) constant velocity relative to Earth's center. (One can juggle balls just as easily on a uniformly moving train or plane as on the ground.) Einstein seized upon this ballistic phenomenon because it was one instance in which at least some "laws" of physics were the same for observers in different states of motion. He dropped the highly specific physical context and made it the foundation of his subjectivistic program for all physics. His SR was defined by these axioms:

1. The restricted principle of relativity: "All laws of nature are the same in all CSs moving uniformly, relative to each other."(39)

2. The law of propagation of light: The velocity of light in vacuum, $C$, is a law of nature and therefore is the same in all CSs moving uniformly relative to each other.

3. The Lorentz transformations: These equations transform measurements taken in one uniformly moving CS to those taken in another.

Simply put, Einstein asserted that all natural laws including the velocity of light can be related to, and are the same for, any observer or arbitrarily chosen CS no matter what its velocity, as long as it's not accelerating (relative to what?). This is subjectivistic, not objectivistic, modeling. Within this system, one cannot ask whether light "really" moves at $c$ relative to every observer or only appears to do so; the observer's appearances and measurements are the only reality. Note that there is no mention in SR's axioms of the Cosmos or of any CS that represents the space or matter of the Cosmos. Einstein thus rid physics not only of Newton and Lorentz's ether, but also of the Cosmos itself. He asserted that all motion was merely relative motion; that there was "no such thing as an independently existing trajectory, but only a trajectory relative to a particular body of reference." ${ }^{\prime(40)}$ "Relativity" meant, quite literally, that all motion and laws of motion were merely relative to any chosen observer or CS, not to the matter and space of any objective or "absolute" Cosmos. Einstein used the CS as a technical tool for modeling the experiences and measurements of any human observer in any state of motion. Indeed, "observer," "frame," and "CS" are essentially interchangeable in the Relativistic literature. Einstein's "relativity of simultaneity" was likewise just the assertion that time, like space, should be treated subjectivistically and therefore was also relative and different for every observer. Contrast this with Aristotle's Cosmic, objectivistic definition of simultaneity: "Those things, moreover, are 'simultaneous' in the unqualified sense of the word which come into being at the same time." we routinely use all the knowledge that we have about distances and light travel times to project a single time frame upon our Cosmos-to determine what moment in our timekeeping system corresponds with the moment that any event "came into being" anywhere in the Cosmos. Observe how we determine when and where a supernova actually occurred. In Relativity, however, time is treated as incorrigibly subjectivistic; it's only what the observer records using his own clock that is supposedly unaffected by his position or motion in any physical Cosmos. Effects may even precede causes for observers in certain states of motion. Relativity's epistemology simply forbids the observer to step out of his own perspective and consider what is really happening, and when, in the Cosmos. 
Is a subjectivistic physics workable? Is all motion merely relative to any arbitrarily chosen frame? Can we actually relate the motion of light and of matter, and their effects, equally well to any human observer or CS? What if instead the evidence indicates that motion is determined by and most simply related to specific, persistent, and massive objects in this Cosmos, such as our Earth, Sun, and stars?

Consider which is more plausible: that light travels at $c$ relative to each and every observer or relative to the immense masses that dot this Cosmos. Consider a light signal and a spaceship leaving Earth in the direction of Alpha Centauri, the nearest star. We have every reason to believe that the light signal would travel the distance in 4.3 years. If the ship arrives later, say, in 5.7 years (or the equivalent greater time in Centaurian units), then the ship's average velocity was $0.75 \mathrm{c}$. What was the velocity of the light signal relative to the ship? Relativity asserts that all light always moves at $c$ in any CS, including that of our spaceship. However, objectively, in this Cosmos, in the local frame of the nearest, greatest masses, the light signal's average velocity relative to the spaceship was only $0.25 c$, not $c$. If the space traveler, using some instruments, actually measured that same light signal or all light as moving at $c$ relative to herself, then we really ought to presume that her instruments were affected by her motion in space so as to produce an illusion of invariant $c$ (Lorentz's theory). If the space traveler nevertheless insists that all light in the Cosmos actually, physically moves at $c$ relative to herself, if she insists that her frame is causative and not the stars' frame, we ought to conclude that she suffers from an anthropocentric delusion similar to that of the Earth-surface observer of the Aristotelian-Ptolemaic system. Notice also that Relativity claims that there can be no relative velocity $>c$. However, if this ship and another ship make this same journey in opposite directions in 5.7 years, then each had an average speed of $0.75 \mathrm{C}$ in opposite directions in the local star frame. Objectivistically, in this region of the Cosmos, their relative velocity throughout was $1.5 \mathrm{c}$, no matter what their instruments measured and no matter how useful their Relativistic assumptions and calculations might be for any other purpose.

Likewise, consider which is more plausible, that matter increases in mass-energy as its velocity approaches $c$ relative to each and every observer or relative to the nearest celestial bodies? Is it more likely that particles in our accelerators require huge voltages to approach $c$ because they are moving at that velocity relative to the human observers in the room or because they are moving at that velocity relative to the adjacent $6 \times 10^{24} \mathrm{~kg}$ Earth? Clearly, the latter is much more likely. Consider also an observer flying past our Solar system at near $c$. According to Relativity, since all bodies in the Cosmos, including our Sun and planets, are moving at near $c$ in his CS, they should appear to him to be much more massive and much closer to each other than they actually are. Since in Relativity all laws of physics are supposed to be the same for him as for an observer at rest relative to our solar system, he should expect gravity to cause these more massive planets to quickly spiral into the more massive and closer Sun and collide with it. In fact, we have no evidence that any motion of any observer has ever affected any other object or process in the Cosmos. Such paradoxes (contradictions) can be evaded only by the introduction of ad hoc fixes resembling the equants and epicycles of the Ptolemaic system. In contrast, an objectivistic physics presumes that the motion of an observer alters only herself and her instruments, not the rest of the Cosmos. Cosmism and objectivism produce no paradoxes and require no apologetics or ad hoc fixes.

Those who believe that Relativity provides an objectivistic model of the Cosmos, even a theory of the physical Cosmos, are thus imprisoned within the contradiction between Relativity's subjectivism and their own natural objectivism. Their tools are inappropriate for their task. They use subjectivistic concepts to describe the objective Cosmos even though this doesn't work and creates paradoxes and confusion. Unaware of the source of the problem, they apply various fixes as needed. Instead of relating motion to any observer as per Relativity, they actually relate motion, including the velocity of light, to Earth, the Sun, and the stars, not realizing that they're contradicting the tenets of their science. To explain inertia or resolve the twin paradox, they introduce a third frame, one that is objective and causative, not realizing that they thus contradict the axioms of Relativity. Caught in an ineradicable confusion between what appears to be (subjectivism) and what is (objectivism), every Relativist creates idiosyncratic answers to such unanswerable questions as whether the Lorentz contraction and time dilation are real or only apparent, whether mass really increases or only appears to do so, whether light really moves at $c$ relative to every observer or only appears to do so, etc. To defend their science from the charge of subjectivism, they produce statements in which Einstein appears to support objectivism and causality, not understanding that Einstein defined "objective" and "reality" exactly as Bishop Berkeley did.

\subsection{General Relativity}

This confusion is only compounded when it comes to GR. GR seems to be objectivistic because it includes the effects of matter and its gravity on the observer's measurements. Does this inclusion make it a model of, or a theory about, the physical Cosmos as distinct from the observer's consciousness? Not at all. In GR, as in Bishop Berkeley's Matrix, mass, inertia, and gravity are merely measured quantities associated with certain alterations in the observer's other sensations and measurements. Their cause is not addressed.

Einstein specified that SR applied only to the measurements of relatively moving, nonaccelerating, nonrotating observers. Relative to what were they not accelerating or rotating? The Cosmos? The matter of the Cosmos? Einstein realized, with disappointment, that SR, like Newton's mechanics, required the existence of a Cosmic, observerindependent inertial frame relative to which the laws of nature applied in a special way. He desired to eliminate this last vestige of the Cosmos from physics. He did not want any 
reference bodies or CSs, including the celestial bodies of the Cosmos, to be given priority over any other CSs. ${ }^{(42)} \mathrm{He}$ did not want the CSs of Earth, the Sun, and the stars to be given priority over the CS of any human observer. I have explained the source of his program. His solution was to "relativize" all motion - to treat inertia and gravity also as merely relative motions. He tried to generalize the principle of relativity to relate all laws of nature to any observer's CS whatever his state of motion. GR remained, like SR, completely subjectivistic.

Serendipity is a common occurrence in the history of natural philosophy. We have often discovered facts or causes accidentally and in spite of our false preconceptions. Einstein, having abandoned Newton's useful but inadequate theory of space and using his method of thinking about what observers in various states of motion would feel and measure, did discover important facts about gravity. Whereas Newton had treated gravity as an occult attractive force proportional to the mass, Einstein built upon Galileo's discovery that gravity's essence was acceleration-it imparted the same acceleration to every test mass, regardless of its weight. Einstein concluded that gravity was just an accelerational field. In one of his classic thought experiments, he reasoned that a blind observer in a large box being accelerated in deep space by a rocket might reasonably conclude that her box was instead suspended motionless in a gravitational field. This led to his principle of equivalence of inertial and gravitational acceleration, ${ }^{(43)}$ which encompassed the fact that, for the observer, these two forms of acceleration felt the same and had similar effects on her measurements. The purely subjectivistic nature of this principle was clearly demonstrated by Einstein's assertion that observers feeling their deceleration on a braking train could legitimately claim that a temporary gravitational field had been created. ${ }^{(44)}$

Einstein used this subjectivistic model of accelerated motion, and the velocity resulting from this acceleration, to predict the effects that gravitational fields should have on the observer's measurements of time and of the motion of light. $\mathrm{He}$ found the non-Euclidean geometries of Gauss and Reimann suitable for this purpose. His Gaussian CS was simply a nonrigid, non-Euclidean geometric model of the observer's space-time measurements. Einstein's generalized principle of relativity asserted that "All Gaussian co-ordinate systems are essentially equivalent for the formulation of the general laws of Nature." ${ }^{(45)}$ Note again that GR retained SR's subjectivistic foundations: absolute $c$ for every observer's CS, time as the observer's clock reading, and distance as the observer's measuring rod. Where SR had related the "laws of nature" only to observers in uniform motion or free fall, GR attempted to relate all "laws of nature" to any rotating, randomly accelerating reference "mollusk." (46) Now just imagine how our solar system, the Cosmos, or any physical phenomenon would appear to, or be measured by, an observer tumbling through space in a ship whose stabilizing jets were firing randomly. Imagine the physical nonsensicality and mathematical complexity of relating the laws of physics to his Gaussian, writhing, mollusk-like CS-this was GR. Would such an observer ever be able to understand the motion of our solar system? In fact, his situation is similar to but much worse than that of the rotating Earthsurface observer. Recall the complexity involved in the Ptolemaic program of describing the planets' motions in the frame of the regularly rotating Earth-surface observer. Recall the immense effort needed, over many centuries, to escape this anthropocentric perspective. In fact, Relativists do not apply the rules of GR to any tumbling CS, but always to the CSs of the celestial bodies of the Cosmos. They give priority to these CSs even though their ideology explicitly denies their priority. They are using a subjectivistic model in an objectivistic way.

Now it is true that gravitational and inertial acceleration are analogous processes and produce very similar effects on the observer. However, the successes of GR do not prove the validity of its epistemology. On the contrary, the facts contradict its assertion that all motion and laws of motion are merely relative to any chosen CS. As Aristotle argued, matter alone creates the accelerational field we call gravity, not one's choice of CS. We also know now that the description of any motion is always uniquely simple in the frame of the nearest celestial body. Describing the motion of light, satellites, atomic clocks, or matter in any other frame(s) introduces unnecessary and sometimes impossible complexity; relating the laws of physics to any other frame produces contradiction. It was just this problem that Copernicus eliminated by choosing the Sun/star frame for the description of the motion of the planets.

Einstein did not succeed in his quest to overturn the Copernican revolution. One cannot drop the context; one cannot remove the Cosmos from physics. One cannot make the physical effects of gravity or forced acceleration disappear or appear just by imagining a CS that is or is not accelerating with the object or observer. Clearly, one cannot maintain that motion is equally well described in any observer's CS and in the Sun/star CS. How did Einstein deal with this contradiction between reality and his subjectivistic method? He simply evaded it. While he agreed with Mach, even after creating $G R$, that there was a unique Cosmic inertial frame related to the matter of the Cosmos that had to be given priority, he didn't realize or admit that this fact contradicted Relativity nor did he go on to interpret this fact and its implications objectivistically. This is the origin of the longstanding controversy about the relationship of Mach's principle to GR. If Einstein had fully accepted Mach's principle, then he would have had to abandon Relativity and create a theory of how matter affected its surrounding inertial space so as to create gravity and define the local and Cosmic inertial frames. He would have had to abandon his subjectivism and create a speculative Cosmic theory of what space and matter were and how they interacted! Such a philosophical conversion was understandably unlikely.

Relativity is not an argument against objectivism in physics - such an argument has not been made since Berkeley. At best, it may be said that Relativity was a temporary 
stage, a haven from our ignorance, a respite from our theoretical difficulties. It allowed us to have a period of "normal science" in which scientists could accumulate facts and apply mathematical formulas without fretting about their ignorance of the causes of things (Kuhn 1962). It was, however, unfortunate that because of Relativity's successes and philosophical ambiguity, it was never understood and was instead elevated as a sublime doctrine and made immune to criticism. Be that as it may, there is now nothing preventing us from approaching the Cosmos objectivistically; and all the evidence encourages us to do so.

\subsection{Quantum Theory}

In contrast to Relativity, the subjectivism of QT is generally recognized, although the nature and implications of its subjectivism and its constructs are not. A leading textbook states,

Quantum mechanics, however, regards the interactions of object and observer as the ultimate reality. It uses the language of physical relations and processes rather than that of physical qualities and properties. It rejects as meaningless and useless the notion that behind the universe of our perception there lies a hidden objective world ruled by causality; instead, it confines itself to the description of the relations among perceptions. ${ }^{(47)}$

Bishop Berkeley would have again been delighted to see that his program for Science was so faithfully instituted. QM is fully compatible with Berkeley's theory that reality is a mental-spiritual matrix through which our minds interact with God and with other minds. Has anyone ever come up with a better theory than Berkeley's for why we experience what we do if there is no "hidden objective world ruled by causality"? In fact, QT and all its associated ideas are products of subjectivistic idealism; they were not and are not a necessary response to the facts.

In 1905, nearly everyone believed that light was composed of physical waves. The wave theory was necessary to explain interference, diffraction, refraction, etc. Thomas Young had directly demonstrated the wave nature of light. However, as we have shown, Einstein was not interested in explaining physical reality. He noted that there were two experimental phenomena, involving high-frequency EM radiation, that were impossible to explain using the conventional wave theory of light and the conventional particle theory of matter: blackbody radiation and the photoelectric effect. Planck had discovered that the interaction of light and matter occurred only in discrete quanta of EM energy. Carrying this analysis to the photoelectric effect, Einstein asserted, "the theory of light that operates with continuous spatial functions may lead to contradictions with observations if we apply it to the phenomena of the generation and transformation of light." He proposed that "the energy from a beam of light is not distributed continuously over larger and larger volumes of space but consists of a finite number of energy quanta, localized at points of space, which move without subdividing and which are absorbed and emitted only as units." ${ }^{(48)}$ Notice the crucial ambiguity here: are these quanta actual physical particles flying through Cosmic space or are they only a subjectivistic construct-an accounting technique for the prediction of observed absorptions and emissions?

Epistemologically, Einstein's light quantum hypothesis was not distinct from his rejection of ether; indeed they were contemporaneous. Obviously, if one's epistemology is subjectivism, if one only models sensations and measurements instead of theorizing about the physical reality that causes them, then one cannot and will not ascribe reality to anything that is not directly evident to one's senses or instruments. One cannot directly perceive light waves or an ethereal medium. One can perceive the digital output of a photomultiplier. Since certain interactions of light and matter have the quality of quantization, one can, for the purposes of calculation and prediction of certain phenomena, treat light mathematically as quanta (photons) that travel from one point to another. Notice that light is not unique in this regard! One can also treat sound as composed of flying "phonons" for the purpose of mathematically describing acoustic-crystalline interactions, but does anyone actually believe that sound is composed of phonon particles flying through a void?! One can also treat heat as a caloric fluid that flows from one place to another, but no one believes in caloric anymore. As with Relativity, the fact that a certain conceptual scheme produces a working mathematical model of our perceptions and measurements in a limited sphere of our limited experience does not imply that the model is a faithful representation of the underlying physical reality. Different conceptual schemes may yield accurate results. All we can say about the photon is just that it is a "convenient fiction" for certain purposes. It is not a physical hypothesis within a physical theory. Indeed, when considered to be a physical particle, the photon produces contradictions ad nauseam. Einstein wanted to believe that it represented some underlying reality, but it so contradicted everything else known about light that Einstein struggled with it to the end of his life.

Here we find again, as with Relativity, that physicists do not sufficiently appreciate the subjectivistic nature of QT. They believe that it tells them something about physical reality. Like Einstein, they confuse photons as a subjectivistic tool of calculation with photons as a physical entity. We can observe this confusion in Richard Feynman's interpretation of Quantum Electrodynamics (QED). ${ }^{(49)}$ In QED, EM radiation sources produce no unobservable physical light waves, nor even any unobservable flying photons, but only wave-like probabilities of observable, countable, quantized, "photonic" absorption/emission events. QED is just the mathematical modeling and prediction of the observable interactions of light and electrons. ${ }^{(50)}$ Feynman understands this to an extent. He realizes that QED accounts only for what is instrumentally observed by us humans and ignores what is not observed. He realizes that it does not attempt to model or to explain the Cosmic entities and processes that produce our experiences; it does not attempt to offer any reasonable 
explanation for why Nature "behaves" as it does. He asserts that the only criterion of a good theory is whether its predictions agree with experiment. However, he also claims that QED is a description of "Nature." Now if by "Nature" he meant only consciousness as per Berkeley, there would be no confusion; but he means more than that, for we find him asserting, "light is made of particles."(51) Realizing that the photon makes no sense as a physical hypothesis, Feynman concludes that Nature is absurd. ${ }^{(52)}$ I have demonstrated that the absurdity stems from the metaphysics and epistemology of QT, not from the Cosmos. Whatever the successes of QED, it does not and cannot require us to believe that light is made of particles or waves, or of anything whatsoever. The question of the physical nature of light-whether it propagates as waves in a physical space or as particles in a void-is not addressed and cannot be discussed within Einstein's subjectivistic physics. This is a question of physical theory and as such requires a Cosmic metaphysics, an objectivistic epistemology, and much theoretical work.

The "collapse of the wave-function," like the "relativity of simultaneity," is just a corollary of subjectivism. Since QT deals only with the observers and their conscious experiences, what causes a probability to become an actuality? Certainly not any underlying physical Cosmos or physical causes! What then? It must be the observer's own act of observing! It must be that her decision to observe or not to observe determines what "reality" will be! It's really quite simple: subjectivism in, subjectivism out. Erwin Schrödinger attempted to show the absurdity of this solipsistic subjectivistic physics with his catin-the-box paradox. He rebelled against the Copenhagen interpretation that held that the observer's knowledge was the only reality. According to it, it is meaningless to ponder whether the atom decayed and whether the cat is dead or alive absent a human observer taking a look. (Compare this to an ancient subjectivistic Zen paradox: Does a tree falling in a forest make a sound if there is no human to hear it?) Schrödinger argued that the Copenhagen Interpretation of QT couldn't be a complete description of reality, but his mere assertion of an objectivistic position was not sufficient to overturn the ideological foundations of QT and all modern physics. We will discuss Einstein's attitude toward QT below.

\section{EINSTEIN'S MATHEMATICAL IDEALISM}

Einstein admitted that in the field of epistemology he had been an "unscrupulous opportunist," resorting to realism, idealism, positivism, and Platonism as the situation seemed to require. ${ }^{(53)}$ We find indeed that Relativity began in pure Berkelian-Machian subjectivism-sensationalism and ended in Einstein's unique brand of subjectivistic mathematical idealism. We will examine how and why this transformation occurred and how Einstein's constructs came to be misinterpreted as physical entities.

Einstein's thinking changed as he aged. As a young man, he frequently repeated statements to the effect that Science was just a subjectivistic endeavor-the "comprehension, as complete as possible, of the connections between sense experiences ..., ${ }^{\prime \prime 54)}$ yet when he was older, he claimed that Science was based on the belief in an "external world independent of the perceiving subject." ${ }^{(55)}$ Had Einstein arrived at a belief in a physical Cosmos? Not quite.

Einstein described this transformation. I quote:

In my younger years, however, Mach's epistemological position also influenced me very greatly, a position which today appears to me to be essentially untenable. For he did not place in correct light the essentially constructive and speculative nature of thought and more especially of scientific thought; in consequence of which he condemned theory on precisely those points where its constructive-speculative character unconcealably comes to light, as for example in the kinetic atomic theory. ${ }^{(56)}$

Mach believed that atoms were just "convenient fictions." Einstein disagreed. He believed that this construct existed, but how? He explained later in the same publication:

Physics is an attempt conceptually to grasp reality as it is thought, independently of its being observed. In this sense, one speaks of "physical reality." (57)

"Being" is always something which is mentally constructed by us, that is, something which we freely posit (in the logical sense)

. The justification of the constructs, which represent "reality" for us, lies alone in their quality of making intelligible what is sensorily given.... (58)

Einstein believed in atoms and his other constructs, but only because these ideas worked to make our experiences intelligible. Einstein reached out to a reality beyond our sensations, but that reality consisted only of more ideas. Beyond reality as it is observed he believed in reality "as it is thought." He did not posit a physical Cosmos, nor did he produce any physical hypotheses. Commenting on Einstein's work, the philosopher Victor Lenzen noted,

Indeed, since the propositions of (Einstein's) physical science are about complexes of sense-impressions, the independent concept of bodily object need not refer to a reality beyond experience ... The term "independence" suggests a realist conception of objects, but in my judgment the term may be applied to a construct of thought which serves to establish order among sense-impressions ... In view of his distinction between thought and sense, Einstein's doctrine has elements in common with the Platonic conception of mathematical objects as exact forms that transcend sense. ${ }^{(59)}$

Einstein fully agreed with Lenzen's characterization of his position. ${ }^{(60)}$ Therefore, he believed that his atoms, photons, absolute $c$, space-time, and "Cosmos" existed; they were a part of "being," but only as ideas that proved useful for organizing our sensations.

Mathematical idealism, like subjectivism, is very old. It 
began with the dualistic theories of Pythagoras and Plato. They considered the physical Cosmos to be a manifestation of sublime mathematical forms. These forms existed independent of the human mind and of physical reality-they were the templates for all existing entities. The actual relationship of these forms to physical reality was highly problematical. In fact, they were linguistic representations ("chair," "tree," etc.) elevated to a sublime existence. Kant's "transcendental idealism" placed the ideals within the human mental structure-as categories by which the mind interpreted reality. Due to their success, Kant presumed that Newton's absolute space and time and their Euclidean geometry were a prioricategories. Einstein improved upon Kant's idealism by insisting instead that the ideals were free creations of the human mind. He even admitted that the reality he ascribed to his constructs was contingent upon their being replaced by better constructs. However, his followers, like all followers, turned his constructs into dogmas.

The evolution of Einstein's constructs from "convenient fictions" to "underlying reality" to "physical reality" is quite understandable. One can choose not to believe in or theorize about a physical Cosmos, but one cannot escape causality. It's obvious that all motions are the result of previous motions, that every state of affairs results from a previous state of affairs, that all effects are caused. The Cosmos is an orderly whole; it is not chaotic; it contains no contradictions. The regularity with which certain events always follow certain other events demands an explanation. Humans are curious creatures, driven by an instinctual desire to explain the causes of things. They cannot be mere subjectivists and phenomenalists, and neither could Albert Einstein. Since Relativity and QT, by their axioms and methods, excluded a physical cause for the regularity of the observed phenomena, he and subsequent physicists had to introduce some other kind of "cause." The mathematical constructs were the only remaining candidate. Subjectivism here, as always, returns to the idealism upon which it is based. ${ }^{(61)}$ If one does not assume the existence of anything apart from one's consciousness, if one arrests knowledge at the mathematical modeling of the contents of one's consciousness, then one is tempted to ascribe reality and even independent existence to one's mathematical constructs. So Einstein's freely created ideas (space-time, mass-energy, absolute $c$, etc.) came to be treated as "absolute" ideals-as the reality underlying our experiences and thus the Cosmos itself. They became "laws of nature" and the "causes" of the phenomena. Thus, many physicists believe that the Cosmos is ultimately created by and ordered by these mathematical "ideas" that are either self-subsistent or emanate from the mind of God. Stephen Hawking admits that he attempts to "know the mind of God" $^{\prime(62)}$ by searching for the mathematical ideas and equations that "produce" everything. Idealism gave us Relativity and QT, and Relativity and QT perpetuate idealism.

Thence arises another layer of confusion; for most "theoretical" physicists are not Einsteinian idealists. They believe in the existence of a physical Cosmos and seek to know the causes of things. They attempt to "explain" the physical Cosmos by reifying Einstein's subjectivistic constructs. I have already discussed the problems inherent in the reification of absolute $c$ and the photon. Let us now consider the reification of the observers" "space-time," "symmetry," "broken symmetry," and "information."

From an objectivistic and Cosmic perspective-if we assume that there is a physical Cosmos that produces our experiences- "time" can only be our way of describing and measuring the pace of physical evolution in the Cosmos-the endless chain of causes and effects. Time is how we measure motion, and motion measures time; they define each other ${ }^{(63)}$ We compare the motion of interest to some regularly repeating motion, such as the ticking of a clock, the vibrations of an atom, or the frequency of light absorbed and emitted by atoms. We now know that an atomic clock moving relative to the nonrotating Earth-centered frame suffers a $2^{\circ}$ Doppler redshift of its atoms' emitted light and that muons' half-lives are increased at high velocity in this frame. Atomic clocks run slower in gravity. Are we justified in calling these specific physical effects a "slowing" of some ideal called "time"? On the contrary, free electrons' frequencies are blueshifted with increased velocity, and pendulum clocks run faster in gravity. Given the facts, shouldn't we just say that certain physical phenomena have an increased duration relative to other physical phenomena in certain circumstances, and then try to discover the physical mechanism involved?

In idealistic Relativity, the subject's clock-time instead becomes an ineffable ideal form-an imaginary fourth dimension added to his idealized Cartesian three-dimensional CS. The observer's CS, his measurements of distance and time, are reified as a four-dimensional space-time model of his experienced world. For Einstein, this four-dimensional geometric description of experience is the underlying "reality" that produces our experiences. Notice that spacetime is not a model of the physical cosmos. It is a model of the observers' experiences-composed of the one thing that different observers can agree upon in Relativity's conceptual scheme: the space-time intervals that they measure between events. Does the measured interval $d s^{2}=c^{2} d t^{2}-\left(d x^{2}+d y^{2}+\right.$ $d z^{2}$ ) represent any Cosmic entity or physical process? No, it does not. Einstein admitted that the chronotropic interval had no physical meaning or significance. ${ }^{(64)}$ What must we say then of Relativity itself? Again, the intervals and the space-time continuum composed of them are subjectivistic, they represent only the measurements made by observers; each observer assuming, anthropocentrically, that light moves at $c$ relative to herself and that her atomic clock's rate is not altered in any way by any motion in any Cosmos. Indeed, Bishop Berkeley would approve. He would say that in spacetime Einstein had discovered the rules by which God coordinates the experiences he feeds to our minds-the rules of the spiritual Matrix. Space-time is objective only in Berkeley and Einstein's sense of the word-as representing some experiences upon which observers can agree. 
Most Relativists, however, are not subjectivistic idealists. They do not realize that space-time is a subjectivistic matrix. They objectify and reify it. Not only do they take it to be a model of the Cosmos itself, but also the physical "cause" of motion and its effects! They mistakenly claim that space-time "causes" light and matter to move as they do; that matter must follow geodesic "curved paths" in space-time and that the curvature of space-time "causes" light and other matter to gravitate toward matter-even though space-time is nothing but a description of events and motion in the observers' CSs, as detailed above. Their confusion is evident when they often say, mistakenly, that light travels at $c$ in spacetime, not relative to every CS, and when they inappropriately drop "time" from "space-time," and speak of "space" alone as if it were an objective Cosmic reality separable from time.

"Symmetry" is also a subjectivistic description that has been transformed into a mathematical ideal and then a physical cause. All laws of nature, and thus everything in the Cosmos, are supposedly derived from the fact that things "look" the same to observers with different points of view, or look the same after undergoing certain movements. We are told that the Cosmos exists as it does only because of "broken symmetry." A respected "theoretical" physicist can claim, without apparent embarrassment, that "the invariance of the laws of nature under the symmetry transformation of resetting our watches is why there is such a thing as energy ... The symmetry among different frames of reference requires the existence of gravity." ${ }^{\prime 65)}$ We have here a language-endowed primate, himself a product of the evolution of the Cosmos, believing that the Cosmos and its phenomena exist and evolve as they do because of the ways that he sees things and counts them! Darwin would be amused.

In QM, the Cosmos is replaced by the observers' "information." There are no physical entities and causes but only the observers' information and the rules that govern the changes in that information. Witness the academic discussions of what happens to matter that falls into black holes-all based upon speculations about what must happen to the observers' information! With QM, mentalistic constructs have replaced physical reality; Mind has replaced Cosmos. We are assaulted with a veritable word salad of mathematical fixes for these subjectivistic models, fixes that are then inappropriately reified as Cosmic entities and causes. There are strings, zeropoint vacuum energy, virtual particles, renormalization, sums over histories, many universes, etc. Modern Cosmologists are in the most uncomfortable position of having to use idealistic Relativity and QM in their attempts to understand the model and explain the physical Cosmos. They are forced to supply additional fixes like dark matter, dark energy, and quintessence that are no different in kind from Ptolemy's equants, epicycles, and deferents. What is most ironic is that all these fixes, all of these hypothesized, unseen entities and causes are exactly the sort of speculative metaphysical objects that Berkeley, Hume, Mach, and the young Einstein sought to purge from Science! In fact, they did achieve their aim-they purged Science of physical hypotheses and filled it instead with idealistic hypotheses. So let us turn to the validity of, and the motivation for, this replacement of the physical with the ideal.

\section{COSMISM OVER IDEALISM}

Since the origin of language, we have struggled to understand the relationship between mind and reality. Since the advent of transcendental religions, we have found it easier, and emotionally more comforting, to believe that our minds participated in some mental-spiritual reality and that the perceived Cosmos was just an aspect of that reality. This metaphysics supported a belief in God, in miracles, and in immortality. Relativity and QM were produced by a series of essentially religious men who knew very little about the workings of the physical world, their bodies, or their brains. It was easy for them to assume that these things were impenetrable mysteries, and to assume that our unique linguistic intelligence and understanding must be supernatural in origin. So this brings us to the root question: Is idealism/spiritualism a defensible theory of the nature of consciousness and its objects? Perhaps it was a few centuries ago, but not anymore. Unlike Ockham, Descartes, Locke, Berkeley, Hume, and Mach, we have some understanding of what matter is, how it evolved into living creatures, how it is organized into nervous systems, and how nervous systems interact with matter and light to produce sensations. Thanks to the work of many scientists and theorists in recent decades, we now know that the simplest, noncontradictory explanation for how we came to exist and why there appears to be a material Cosmos is that there is a material Cosmos from which we humans evolved. Our sensations are the product of the interaction of our nervous system with the rest of the Cosmos. Certain kinds of physical phenomena cause physical changes in our sensory receptors, and these are transduced into neural signals that are processed by neurons in specific areas of our brains. Our sensory system is an evolutionary adaptation, allowing us to produce a sophisticated and highly useful representation of the physical Cosmos. The entire process is a cascade of identifiable causes and effects-physical, biological, neurological, and linguistic.

We agree with Berkeley that we have no direct, unmediated knowledge of any material cosmos-all our knowledge must originate in our sensations and experiences. However, does this fact force us to limit our knowledge to what is given in experience? Of course not! We have a linguistic intelligence that allows us to theorize about the physical cosmos from which we evolved and with which our nervous systems interact! We can agree with Einstein that all the "constructs" we create to explain our experiences are tentative and may one day be replaced with better constructs. However, nothing prevents us from producing constructs that are objectivistic physical hypotheses. We further realize that all our understanding is "theory soaked," involving that cognitive leap from experiences to causes. Think about how Copernicus and Darwin revolutionized our thinking-not with new facts, but with powerful explanatory theories that now form the 
foundations of our understanding. Cosmic philosophy is just theory writ large; its essence is to reach beyond our experiences to find the best explanation for our experiences. Given all that we know today, the best explanation is that there is a physical Cosmos from which we evolved and with which our minds and instruments interact.

An important recent advance in this philosophical endeavor is the availability of powerful naturalistic theories of the evolution of language and consciousness. ${ }^{(66)}$ We can now say that the best theory to explain our unique mental abilities is that we are intelligent primates who acquired language through a natural process, an evolutionary adaptation unique to the humanoid species. Our increasingly sophisticated use of language, including writing, produced a new kind of information-processing ability-the ability to create and criticize theories about the Cosmos and ourselves. Language allows us to understand the Cosmos and ourselves in a way that other animals (and humans without language) cannot. Other animals are surely conscious, but only humans have language-enhanced consciousness. We no longer need the idealist/spiritual hypothesis to explain our mental life, and thus there is no longer any defensible rationale for restricting our knowledge to the mere description of our conscious experience. The arguments of Descartes, Locke, and Berkeley are therefore irrelevant. Ignorance of causes is no longer an excuse to regress to idealism and subjectivism. The foundation of Relativity and QT is obsolete.

Against idealism of every kind, I assert that Cosmism is the superior foundation for our theories of what exists and of how we experience it. Let me summarize the current state of Cosmic theory:

1. The Cosmos exists and evolves. It is probably cyclical (-Bang-Crunch-Bang-). It has produced on our Earth these hierarchical levels of complexity: Physico-chemical, Biological, Neuropsychological (animals only), and Linguo-philosophical (humans only). At each higher level of complexity, preexisting entities and processes combine and interact in new ways to produce new entities and processes.

2. The nervous system of humans, like that of all other animals, has evolved via natural selection to capture certain Cosmic motions and to synthesize these into mental representations of Cosmic entities and events.

3. As the result of genetic change through natural selection, humans acquired the ability to use language-an advanced information-processing tool.

4. Humans learned to use language not only to name and describe things, but also to create theories of the underlying and unapparent causes of the entities and events that they perceived. This ability to theorize evolved from mythology to philosophy.

5. Humans' increasingly sophisticated use of language produced a sophisticated knowledge and awareness of self and Cosmos-the phenomenon of language-enhanced consciousness.
I join Aristotle and Francis Bacon in rejecting idealism, and mathematical idealism in particular. Our mental life is a virtual reality created by, and dependent upon, our brains - a kind of software run on the brain's hardware. Logic and mathematics are powerful intellectual tools that humans invented for the purpose of better representing fundamental Cosmic relationships and quantities, just as we invented theories to explain the causes of things. Logic and mathematics are software that we invented to increase the efficacy of our brains. They represent certain aspects of Cosmic entities and processes. They are no substitute for physical theory. They are produced by a mental process of abstractionignoring all attributes of Cosmic entities and processes except quantity, extension, shape, movement, and implication. Logic is the bridge between Cosmic causality and our linguistic representations of same. Mathematics is the bridge between Cosmic entities and processes and our quantitative representations of same. Mathematics adds quantitative precision to our observations, predictions, and theories. Logic and mathematics work because the Cosmos is a stable, interacting system that evolves through cause and effect and apparently has some discrete smallest "Quantum" (at the Planck scale?) that determines the size and character of all composite entities and processes. Cosmic logic and mathematics work to the extent that they accurately represent the underlying physical substance(s) and processes. Imaginary logic and mathematics, on the other hand, are creations of the human mind that have no necessary relationship to Cosmic reality. The large role of mathematics in physics as compared with other sciences stems from the simplicity and uniformity of its objects and their interactions. As one moves up the hierarchy of Cosmic organization to geology, biology, psychology, and sociology, mathematics becomes all the more dependent upon, and subservient to, causal theory.

\section{CONCLUSION}

Modern physics is now in a philosophically immature state analogous to that of the Ptolemaic Cosmology before Copernicus and the creationist biology before Darwin, and for the same reasons. Like them, Relativity and QT contain many facts and correlations; they accurately describe and predict the observer's experiences in our limited experience, but they fail to provide any working theory of the causes of things. Like them, Relativity and QT substitute anthropocentric, mentalistic ideals for natural causes. Like them, Relativity and QT's errors are philosophical. As pre-Copernican astronomy merely described motion in the Earth-observer's frame, so Relativity merely describes motion in any observer's frame-as if the observer were not really moving in a physical Cosmos. As pre-Darwinian biology merely described the species without trying to explain their genesis or alterations (they were just created that way by God), so Relativity and QT merely describe and predict observed entities and events without trying to explain their natural causes (God made them so or they just are that way). Since Einstein's physics precludes any naturalistic explanation of the Cosmos, it 
stands in the way of our understanding of the cosmos and of ourselves. Theoretical physics thus awaits a philosophical revolution no less profound than those begun by Copernicus and Darwin.

Let no one ever again assert that Relativity and QT are physical theories of the Cosmos. Let no Relativist or Quantum Theorists mislead themselves or others into believing that they're attempting to understand and explain the physical Cosmos. Instead, let us use our knowledge of Cosmic phenomena to create objectivistic models and physical theories. Let us use our minds to reach beyond consciousness to the Cosmos; beyond Relativity and QT to Cosmic theory.

As Francis Bacon observed, civilization is now older than ever before. We know more about the Cosmos and ourselves than all previous generations. We should not blindly venerate old ideas from our civilization's youth. We should not slavishly follow ideas that are hundreds or thousands of years old. We should criticize the ideas that form the basis of our thought and our sciences and replace them with better ideas. We should admit our ignorance of the causes of things. We should reach beyond our experiences and create theories of the physical nature and mechanisms of space, light and matter. We should incorporate the latest data and the truths contained in our idealistic models into a new theory of the Cosmos. This task is Cosmic philosophy-the openended, antidogmatic, uninhibited search for the truth. A philosophical Cosmology is needed to inform and criticize the specialized sciences-creating a feedback loop that is crucial to the advance of knowledge. As Francis Bacon put it long ago,

Yet this very philosophy it is that ought to be esteemed the great mother of the sciences. For all arts and all sciences, if torn from this root, though they may be polished and shaped and made fit for use, yet they will hardly grow ... let no man look for much progress in the sciences-especially the practical part of them-unless natural philosophy be carried on and applied to particular sciences, and particular sciences be carried back again to natural philosophy. ${ }^{(67)}$

\section{APPENDIX: EINSTEIN ON THE COPENHAGEN INTER- PRETATION OF QUANTUM THEORY}

This analysis allows us to answer a vexing question: Why did Einstein reject the QM that he helped to create? I will demonstrate that the Einstein-Bohr debate was a clash of two varieties of idealism.

At the age of 70, Albert Einstein asked Abraham Pais whether he really believed that the moon existed only if he looked at it. ${ }^{(68)}$ Thus did Einstein express his discomfort with the solipsism of Bohr and Heisenberg. The solipsist holds that the world is only her experience-nothing and no-one exist unless she is experiencing them. Bishop Berkeley was not a solipsist. He would have agreed with Einstein that the moon did exist whether or not any individual human being's consciousness was attending to it-because it was a feature of God's spiritual Matrix. Einstein, on the other hand, could only argue that the Moon existed because its continued existence when not being observed was a necessary and useful mental construct to explain why all humans shared this experience. The weakness of this argument, and the fact that neither Einstein nor anyone else succeeded in finding any "construct" that could explain the reality underlying the observer's experience of quantized events, allowed Bohr and Heisenberg to determine the future of physics.

Einstein rejected Bohr and Heisenberg's probabilistic Copenhagen interpretation of QM as an incomplete description of nature because it stopped at the description and prediction of sensations and did not attempt to discover the "constructs" - the underlying reality - that produced the sensations. The problem he had with QM's $\Psi$ function was not its subjectivism, but its solipsism and indeterminism. Einstein sought to construct a model of a deeper level of "reality" at which quantized events were determined. He insisted that solipsism was untenable and could be overcome by retaining the distinction between "sense impressions on the one hand, and mere ideas on the other." Einstein insisted that we must admit that our sense impressions are conditioned by a "subjective" and by an "objective" factor, for the simple reason that this distinction works to make the contents of consciousness "intelligible." The "objective factor" is the totality of such concepts and conceptual relations as are thought of as independent of experience, viz., of perceptions. He stated that insofar as physical thinking justifies itself by its ability to grasp experiences intellectually, we regard it as "knowledge of the real." ${ }^{\prime 69)}$ He saw no reason why we should give up this program in the "microscopic" when it worked so well in the "macroscopic," as the two realms were so obviously interrelated.

So Einstein's problem with QM was that it represented only the observer's experience and did not attempt to represent the "real factual situation" within the Matrix. It did not go beyond reality as experienced to "reality as thought," as did GR's field equations. Since QM merely described the statistical relationship between the observer's earlier and later experiences and measurements, it left reality wholly dependent on the observer's acts. It had God playing dice with the observer instead of running an organized universe according to determinative rules. As he said, "I still believe in the possibility of giving a model of reality which shall represent events themselves and not merely the probability of their occurrence." ${ }^{(70)}$ He believed that we should seek a deeper-lying theoretical framework that permits the description of phenomena independent of any specific observer or experimental conditions. He wanted a "theory" that described the underlying Matrix of Quantum events as a system resulting from continuous functions of space according to differential equations. In essence, he wanted a deterministic field theory of light and matter's interactions that resembled and was compatible with his GR. He spent the last decades of his life in a futile search for this unified field theory.

Received 25 June 2002. 


\begin{abstract}
Résumé
Albert Einstein était un idéaliste subjectiviste et mathématique. Son étude de la physique consistait en des modèles mathématiques provenant d'idées de ses sujets-de ses sensations et de ses mesures. Sa "réalité objective" était réalité intersubjective-les expériences sur lesquelles les observateurs peuvent concéder. Ses causes étaient d'idées construites pour systématiser les expériences des observateurs. Il modelait la réalité comme elle étant éprouvé et pensé, et non pas comme elle était. Il modelait la connaissance et non le cosmos. Si nous voulions comprendre le cosmos comme tel, comme un système physique dans lequel nous et nos connaissances ont évolués, nous devons alors bâtir à partir d'une philosophique différente. Nous devons créer des hypothèses et des théories sur ce qui existe et sur les façons celles-ci interagissent avec nos esprits et nos instruments. Il faut aller au-delà de la connaissance du cosmos, au-delà de la relativité et la théorie quantique jusqu'à la théorie cosmique.
\end{abstract}

\section{Endnotes}

I I capitalize "Relativity," "Quantum Theory," and "Science" because they are the proper names of dominant, unexamined ideologies-fixed systems of beliefs concerning what exists and how we should study it. I do not capitalize the well-studied and defined "isms" of philosophy like realism, idealism, etc. I do not capitalize "philosophy" because it is the open-ended search for knowledge and understanding, not an ideology. I use "Cosmos" as the proper name for everything that exists: space, matter, life, behavior, consciousness, and ideas. I choose to capitalize it and the proper names of other significant entities in our existence, such as "Earth" and "Sun." I use "Quantum Theory" as the more general term designating the idealistic approach to the microcosm initiated by Einstein in 1905 with his "light quantum" construct. I use "Quantum Mechanics" to designate the now-dominant version of QT based upon the solipsistic idealism of Bohr and Heisenberg.

\section{References}

1. Albert Einstein, Ideas and Opinions (Crown Publishers, Inc., New York, 1954), p. 50.

2. Ayn Rand, Philosophy: Who Needs It (The Bobbs-Merrill Co., Inc., New York, 1982), p. 29.

3. Isaac Newton, Mathematical Principles of Natural Philosophy (1686), Definitions, Scholium, para. 9.

4. Albert Einstein and Leopold Infeld, The Evolution of Physics (Simon and Schuster, New York, 1938), p. 224.

5. Plato, Phaedo, 100.

6. Sextus Empiricus, Outlines of Pyrrhonism, I. 216 sq.

7. Aristotle, Metaphysics, Bk. IV, Chap. 5, 1010b, 35.

8. Ibid., Chap. 6, 1011a, 18.

9. Idem., Physics, Bk. IV, Chap. 1, 208b, 14-24.

10. Isaac Newton, Ref. 3, para. 8.

11. Ernest A. Moody, "William of Ockham," in The Encyclopedia of Philosophy, lst edition (Macmillan, Inc., New York, 1967).

12. René Descartes, Meditations on First Philosophy (1641) Med. I, para. 10.

13. Ibid., Med. IV, paras. 5, 9.

14. John Locke, An Essay Concerning Human Understanding (1690) Bk. II, chap. XXIII, para. 15.
15. Ibid., para. 29.

16. George Berkeley, Principles of Human Knowledge (1710) body, para. 18.

17. Ibid., para. 6.

18. Ibid., para. 26.

19. Ibid., para. 94.

20. Idem, An Essay Towards a New Theory of Vision (1709).

21. The Matrix (Warner Bros., 1999).

22. George Berkeley, Principles of Human Knowledge, paras. 92, I17.

23. Ibid., para. 66.

24. Ibid., para 106.

25. Ibid., para. 112 .

26. Ibid., para. 98.

27. David Hume, An Enquiry Concerning Human Understanding (1748), Sect. V, pt. II, para. 11.

28. Ibid., Sect. IV, part I, para. 12.

29. Peter Alexander, "Mach, Ernst," in Ref. 11.

30. Ernst Mach, Analysis of Sensations (Translated by C.M. Williams and Sydney Waterlow, Open Court Publishing, La Salle, IL, 1914), p. 12.

31. Ernst Mach, The Science of Mechanics (Open Court Publishing Co., La Salle, IL, 1960), p. 283.

32. Ibid., p. 293.

33. Albert Einstein, letter to M. Besso, March 6, 1952; found in Albert Einstein-Michele Besso Correspondence 1903-1955 (Hermann, Paris 1972), p. 464.

34. Idem., The Meaning of Relativity (Princeton University Press, Princeton, NJ, 1922), p. 2.

35. Ref. 1, p. 50.

36. Ibid., p. 262.

37. Ibid., p. 274.

38. Ibid., p. 363.

39. Ref. 4, p. 186.

40. Albert Einstein, Relativity, The Special and General Theory (Crown Publishers, Inc., New York, 1961), p. 10.

41. Aristotle, Categories, Chap. 13, 15a, 12.

42. Albert Einstein, Ref. 40, p. 71.

43. Ibid., p. 70.

44. Ibid., p. 70.

45. Ibid., p. 97.

46. Ibid., p. 99. 
47. Robert Eisberg and Robert Resnik, Quantum Physics (John Wiley \& Sons, Inc., New York, 1974), p. 88.

48. Albert Einstein, Ann. Phys. xvii, 132 (1905).

49. Richard P. Feynman, QED (Princeton University Press, Princeton, NJ, 1985).

50. Ibid., p. 14.

51. Ibid., p. 15

52. Ibid., p. 10.

53. Albert Einstein, in Albert Einstein: Philosopher-Scientist, edited by P.A. Schilpp (Tudor, New York 1949), p. 684.

54. Ref. 1, p. 293.

55. Ibid., p. 266.

56. Ref. 53, Autobiographical Notes, p. 21.

57. Ibid., p. $8 \mathrm{I}$.

58. Ibid., Reply to Criticisms, p. 669.

59. Victor Lenzen, in Ref. 53, Einstein's Theory of Knowledge, pp. 366-367.

60. Ref. 53, Reply to Criticisms, p. 683.

61. Karl Popper, Realism and the Aim of Science (Rowman and Littlefield, Totowa, NJ, 1956), p. 103.

62. Stephen W. Hawking, A Brief History of Time (Bantam Books, New York, 1988), p. 175.

\section{Henry H. Lindner}

RRl Box 381-L

Falls, Pennsylvania 18615 U.S.A.

e-mail: hlindner1@yahoo.com
63. Aristotle, Physics, Bk. IV, 220b, 15

64. Albert Einstein, quoted by T. Levi-Civita, Nuovo Cimento 13, 45 (1936).

65. Steven Weinberg, Dreams of a Final Theory (Pantheon Books, New York, 1992), pp. 139, 144.

66. Julian Jaynes, The Origin of Consciousness in the Breakdown of the Bicameral Mind (Houghton Mifflin Company, Boston, 1976); Derek Bickerton, Language and Species (The University of Chicago Press, Chicago, 1990); Merlin Donald, Origins of the Modern Mind (Harvard University Press, Cambridge, MA, 1991); Daniel C. Dennet, Consciousness Explained (Little, Brown and Company, Boston, 1991): John McCrone, The Ape That Spoke (William Morrow and Company, Inc., New York, 1991).

67. Francis Bacon, Novum Organum (1620), Sects. 1xxix and lxxx.

68. Abraham Pais, 'Subtle is the Lord...' The Science and the Life of Albert Einstein (Oxford University Press, Oxford and New York, 1982), p. 5.

69. Ref. 53, Reply to Criticisms, p. 674.

70. Abraham Pais, Ref. 68, p. 460, quoting Einstein's 1933 Spencer Lecture. 